\section{Auch geringer Alkoholkonsum kann riskant sein}

Seit Langem ist bekannt, dass Alkohol das Risiko für ein Ösophaguskarzinom erhöht. Unklar war allerdings bisher, ob auch die Menge des konsumierten Alkohols von Bedeutung ist.

is jetzt fehlten vor allem Daten zum Einfluss des Alkoholkonsums auf das Risiko für ein Plattenepithelkarzinom des Ösophagus (ESCC) bei Personen, die nur wenig Alkohol trinken, nie geraucht haben oder aus asiatischen Ländern stammen. Bei Asiaten kommen einige Hochrisiko-Polymorphismen in Genen des Alkoholmetabolismus häufiger vor. In einer systematischen Übersicht und Metaanalyse von 40 Fallkontroll- und 13 Kohortenstudien wurde unterschieden zwischen:

_ geringem Alkoholkonsum ( $\leq 12,5 \mathrm{~g} /$ Tag),

_moderatem Alkoholkonsum (> 12,5 $<50 \mathrm{~g} / \mathrm{Tag}$ ) und

— hohem Alkoholkonsum ( $\geq 50 \mathrm{~g} / \mathrm{Tag}$ ).

Das Risiko für die Entwicklung eines ESCC war umso höher, je mehr Alkohol die Studienteilnehmer tranken. Das relative Risiko (RR) betrug im Vergleich zu Personen, die überhaupt keinen oder nur gelegentlich Alkohol tranken: _bei geringem Alkoholkonsum 1,38 (adjustierte Studien mit Berücksichtigung von Alter, Geschlecht und Nikotinkonsum) bzw. 1,35 (prospektive Studien),

_bei moderatem Alkoholkonsum 2,62 bzw. 2,15 und

_bei hohem Alkoholkonsum 5,54 bzw. 3,35 .

Auch bei Personen, die nie geraucht haben, steigerte moderater bzw. hoher Alkoholkonsum das Risiko für ein ESCC (RR: 1,54 bzw. 3,09).

Nur in asiatischen Ländern ging ein geringer Alkoholkonsum mit einem erhöhten Risiko für ein Ösophaguskarzinom einher: Das relative Risiko betrug hier 1,52 bzw. 1,89 , in allen anderen Regionen dagegen nur 1,28 bzw. 1,03; die Signifikanz wurde nur in den adjustierten Studien in Asien erreicht.

Fazit: Diese Metaanalyse ergab, dass moderater Alkoholkonsum das Risiko für ein ESCC verdoppelt, und zwar sowohl in

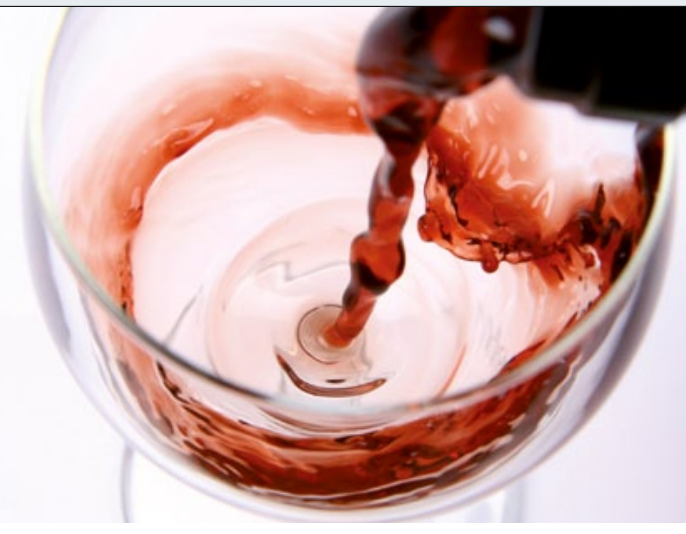

Zwei bis drei Gläser Wein pro Tag reichen schon aus, um das Risiko für ein Plattenepithelkarzinom der Speiseröhre zu erhöhen.

Asien als auch in nicht asiatischen Ländern. Hoher Alkoholkonsum geht sogar mit einem 3,5-fachen Risiko einher. Geringer Alkoholkonsum scheint das Risiko dagegen nur in Asien zu steigern. Vermutlich sind Menschen mit bestimmten Genotypen, die vorwiegend in Asien und seltener in westlichen Regionen vorkommen, empfindlicher gegenüber leichtem Alkoholkonsum.

Judith Neumaier

Islami F et al. Alcohol drinking and esophageal squamous cell carcinoma with focus on light-drinkers and never-smokers: a systematic review and meta-analysis. Int J Cancer. 2011;129(10):2473-84.

\title{
Radiochemotherapie, Operation oder beides?
}

In einer Metaanalyse ging es um die Frage, was eine neoadjuvante Radiochemotherapie bei Patienten mit resezierbarem Plattenepithelkarzinom des Ösophagus bringt und ob eine alleinige Radiochemotherapie ausreicht.

S tandard beim resezierbaren Plattenepithelkarzinom des Ösophagus ist die Resektion mit Lymphadenektomie. Die meisten Patienten erhalten vorher eine Chemotherapie, z. T. mit zusätzlicher Bestrahlung. Allerdings sprechen nur 40-50\% der Patienten auf die neoadjuvante Therapie an. Dem stehen die Nebenwirkungen und die Verzögerung der Operation gegenüber. Als weitere Strategie kam in den vergangenen Jahren immer wieder die alleinige (definitive) Radiochemotherapie mit Verzicht auf die Operation zum Einsatz. Eine Metaanalyse über 20 kontrollierte, randomisierte Studien verglich nun die verschiedenen Strategien.

Dabei zeigte sich, dass die neoadjuvante Radiochemotherapie (RCT) zu einem

signifikanten Vorteil hinsichtlich des Gesamtüberlebens im Vergleich zur alleinigen Operation führte (Hazard Ratio [HR] 0,81; $\mathrm{p}=0,008)$, nicht aber die neoadjuvante Chemotherapie ( $\mathrm{HR} 0,93 ; \mathrm{p}=$ 0,368). Sowohl RCT als auch Chemotherapie steigerten die Wahrscheinlichkeit einer R0-Resektion signifikant um 15 bzw. $16 \%(p=0,043$ bzw. $p=0,006)$. Die neoadjuvante Therapie hatte keinen signifikanten Einfluss auf die Morbidität (RCT: HR 0,94; $\mathrm{p}=0,363$; Chemotherapie: HR $1,03 ; \mathrm{p}=0,638)$ und die 30-Tages-Mortalität (RCT: HR 1,46; $\mathrm{p}=0,116$; Chemotherapie: HR 1,04; $\mathrm{p}=0,810$ ).

Beim Vergleich einer definitiven Radiochemotherapie versus Operation mit oder ohne vorheriger RCT ergab sich kein ein- deutiger Sieger. Die therapiebedingte Mortalität war bei operierten Patienten signifikant höher als bei Patienten, die lediglich eine RCT erhalten hatten (HR $7,60 ; p=0,007)$. Dennoch war insgesamt kein Überlebensvorteil durch eine alleinige RCT nachweisbar. Hinsichtlich der Gesamtmorbidität zeigte sich ein Trend zugunsten der Operation mit vorhergehender neoadjuvanter RCT.

Fazit: Bei Patienten mit resezierbarem Plattenepithelkarzinom des Ösophagus kann die neoadjuvante RCT das Leben signifikant verlängern, ohne dass die Morbidität zunimmt. Eine alleinige RCT hat hinsichtlich des Überlebens keinen Vorteil im Vergleich zu anderen kurativen Strategien.

Judith Neumaier

Kranzfelder M et al. Meta-analysis of neoadjuvant treatment modalities and definitive nonsurgical therapy for oesophageal squamous cell cancer. Br J Surg. 2011;98(6):768-83. 